\title{
Biomedical Applications of Chitosan and its Derivatives - A Review
}

\author{
N. Sundaramoorthy ${ }^{1}$, G. Ramya ${ }^{2}$, A. Lathifa Thasneem ${ }^{2}$, D. Kaviarasi ${ }^{2}$, \\ J. Hemapriya ${ }^{3}$, R. Aswini ${ }^{4}$ and S. Vijayanand ${ }^{2} *$ \\ ${ }^{1}$ Department of Microbiology, KMG College of Arts and Science, \\ Gudiyatham, Tamilnadu, India \\ ${ }^{2}$ Department of Biotechnology, BRT Lab, Thiruvalluvar University, Vellore, Tamilnadu, India \\ ${ }^{3}$ Department of Microbiology, DKM College for Women, Vellore, Tamilnadu, India \\ ${ }^{4}$ Department of Biotechnology, DG Vaishnav College, Chennai, Tamilnadu, India \\ *Corresponding author
}

\section{A B S T R A C T}

\section{Keywords}

Antimicrobial activity, Chitin, Chitosan, Drug targeting, Wound healing

Article Info

Accepted:

25 December 2020

Available Online:

10 January 2021
Chitosan is a deacetylated compound of chitin which is a polysaccharide extracted from the shells of crustaceans, molluscs, fungi and other organisms. Chitosan is made up of copolymers containing glucosamine and $\mathrm{N}$ acetylglucosamine. They are derived by three methods from chitin viz, deproteination, demineralization and deacetylation. It is a basic polysaccharide and has several advantages such as photoelectric behaviour, ability to form films, metal chelation, optical and structural characteristics. These abilities of chitosan made them a perfect candidate for various applications. Chitosan is used in various fields such as cometics, agriculture, food, paper industry and also in fabrics. Besides these, they are widely used in biomedical applications due to its flexibility to be moulded into any shapes and more importantly its non toxic nature. In the field of biomedicine, Chitosan are known for their best anti microbial, anti oxidant, anti cancer and wound healing abilities and it is also used as drug delivery systems for treating several diseases. The present review deals with the biomedical applications of chitosan.

\section{Introduction}

Chitin is the second most extensively spread natural polysaccharides on earth and it is made of $\beta(1 \rightarrow 4)$ linked 2-acetamido-2-deoxy $\beta$-DGlucose. Though chitin is not produced in organisms containing cellulose, it is considered as a cellulose derivative since it resembles structure of cellulose with an exceptional acetamide groups at $\mathrm{C} 2$ positions (Dutta et al., 2003). The $\mathrm{N}$ deacetylation of this chitin at varying extent results in the 
production of chitosan. Chitosan was discovered by Rouget in 1859 ostensibly when he was working with chemical and mechanical nature of chitin (Au natural herbals, 2001). Chitosan is a linear polymer of $\alpha \quad(1 \rightarrow 4)$ linked 2-amino-2-deoxy- $\beta$-D-Glucopyranose (Dutta et al., 2003). The structure of chitin and chitosan is given in Fig 1 and Fig 2. The production of chitosan from chitin follows four steps such as Deproteinization, Demineralization, Decolouration and Deacytelation (Dutta et al., 2003). During these processes chitosan undergoes various transformations of which $\mathrm{N}$ acetylation and Schiff's base reaction are influential. The deacetylation causes chitosan highly basic and soluble in dilute aqueous acidic medium. In addition, they produce various derivatives on reacting with different group of chemicals such as aldimines, ketimines, $\mathrm{N}$ - carboxymethyl chitosan, N-carboxybenzy chitosan etc (Hirano 1997; Sashiwa and Shigemasa 1999; Kurita 1998).

Chitosan has advantageous chemical characters such as reactive amino and hydroxyl groups and chelates many transitional metal ions. In addition to its chemical characteristics it has varying biological advantages such as natural, safe, non toxic, biodegradable, biocompatible and readily binds to mammalian and microbial cell aggressively (Kumar 2000; Dutta et al., 2003; Balmayor et al., 2011). This biological and chemical characteristic along with its cheap cost makes them a perfect candidate for wide variety of applications such as food industries, cosmetics, pharmaceutical industry and in clinical treatments (Kumar 2000). In the present review, the biomedical applications of chitosan are discussed.

\section{Biomedical applications of chitosan}

Chitosan possess several biomedical applications such as antimicrobial activity, antioxidant activity, burn treatments, opthalmology etc. which are discussed as follows:

\section{Antimicrobial activity}

The recent past has been concentrating much on the antimicrobial activity of chitosan and its derivatives (Khanfari et al., 2008; Limam et al., 2011). Two mechanisms were proposed for chitosan exhibiting its antimicrobial i) Interfering with the bacterial metabolism by electrostatic stacking of polycationic chitosan (Chung et al., 2004; Je and Kim 2006) ii) Blocking the transcription of RNA to DNA (Liu et al., 2001). However, the antimicrobial activity of chitosan is highly attributed to its degree of acetylation and molecular weight (Acharya et al., 2005). A study on antimicrobial activity of chitosan prepared from shrimp shell waste showed that chitosan exhibited bacteriostatic activity on the three microorganisms viz, Escherichia coli, Pseudomonas aeruginosa, Staphylococcus aureus (ATCC 43300 and 25923), Bacillus subtilis, Bacilus cereus, Vibrio cholerae, Shigella dysenteriae, Enterobacter agglomerans, Prevotella melaninogenica and Bacterioides fragilis (Benhabiles et al., 2012).

Chitosan with molecular weight ranging from 22 to $846 \mathrm{KDa}$ in combination with most active monoterpenes had better antibacterial activity on bacterial species such as Agrobacterium tumefaciens, Erwinia carotovora, Corynebacterium fasicans and Pseudomonas solanacearcum (Badawy et al., 2016). Commercial chitosan in its gel form has exhibited better antimicrobial activity against Staphylococcus aureus while the gel was less sensitive to E. coli (Goy et al., 2016). Chitosan along with Polyvinyl alcohol doped with leaf extract of Hibiscus sabdariffa showed better antimicrobial activity for Escherichia coli, Pseudomonas aeruginosa, Staphylococcus aureus, Bacillus subtilis and 
Candida albicans (Abdelghany et al., 2019). In this way, chitosan has explored and still studied with its varying derivatives and complexes for antimicrobial activity.

\section{Antioxidant activity}

The increasing interests in finding natural antioxidants to protect human body from oxidative stress have resulted in wide search for all natural resources (Kinsella et al., 1993). Among which chitosan is a natural way of obtaining antioxidants from huge and cheap sources such as shrimp shell, crab shell waste etc.

Therefore, studies on antioxidant activity of chitoan have also extensively made to use them for the purpose. One such study is the enzymatic functionalization of chitosans with flavanoids and exploring its activity for its antioxidant activity. The study showed that the antioxidant activity of chitosan increased with the type of flavanoids used (Sousa et al., 2009). This study confirms the ability of chitosan to bind with other molecules thus indicating its flexibility and also its potential antioxidant activity (Rajalakshmi et al., 2013).

In another study chitosan extracted from shrimp skeleton was found to have better antioxidant activity. Film forming hydrosols formed from the Chitosan/Hydroxypropyl methylcellulose (CH/HPMC) studied for its antioxidant activity showed the efficiency of $\mathrm{CH} / \mathrm{HPMC}$ in free radical scavenging effect and ferric reducing antioxidant power (Zimoch-Korzycka et al., 2016).

Similarly, the antioxidant effect of chitosan was found to be improved by chemical modifications with coumarin (Li et al., 2019). Chitin extracted from Polybius henslowii, a swimming crab was acetylated to produce chitosan oligosaccharides. These chitosan oligosaccharides were found to have better radical scavenging activity, thus better antioxidant activity (Avelelas et al., 2019).

\section{Antitumor activity}

One among the biomedical activities of chitosan is their antitumor activity. The antitumor activity of chitosan is attributed to its membrane disrupting potential and apoptosis inducing activities (Qi et al., 2005). Antitumor activity of chitosan experimented on cancer lines such as Sarcoma-180 and mouse hepatoma $\mathrm{H} 22$ showed that it has great effect on killing cancer cells (Qi et al., 2006).

In another study, the drug used for treating cancer called as mifepristone was loaded on chitosan nanoparticles and studied for its antitumor activity in Human lung adenocarcinoma A549, HELA cell line, Human endometrial carcinoma RL95-2 and Human liver hepatocellular carcinoma HepG2.

Studies revealed that the chitosan loaded with mifepristone showed better anti cancerous activity even in small amounts (Zhang et al., 2016).

In another study, chitosan that are commercially available and those synthesized from mayfly was experimented for its antitumor activity on L929 fibroblast and three different cancer cell lines such as HeLa, A549, and WiDr. The study revealed that the chitosan derived from mayfly had better antitumor activity at a concentration of 500 $\mu \mathrm{g} / \mathrm{L}$ and below $250 \mu \mathrm{g} / \mathrm{L}$ the molecular weight played a major role than the concentration (Tan et al., 2018). Similarly, study on chitosan based $\beta$ ketosulfan derivatives on cancer lines such as colon carcinoma (HCT), liver hepatocellular carcinoma (HEPG2), and breast carcinoma (MCF-7) was found to have high efficiency with lower concentrations (Alamry et al., 2018). 
Fig.1 Structure of chitin (De Alvarenga 2011)

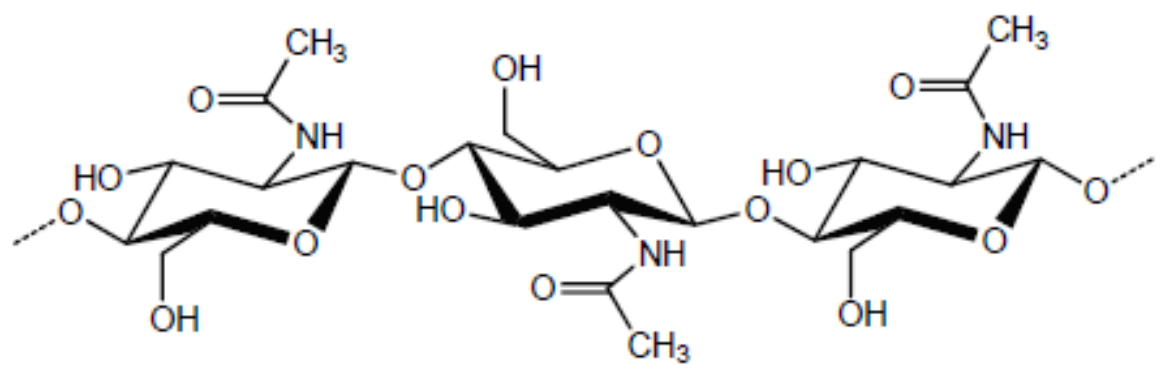

Fig.2 Structure of Chitosan (De Alvarenga 2011)

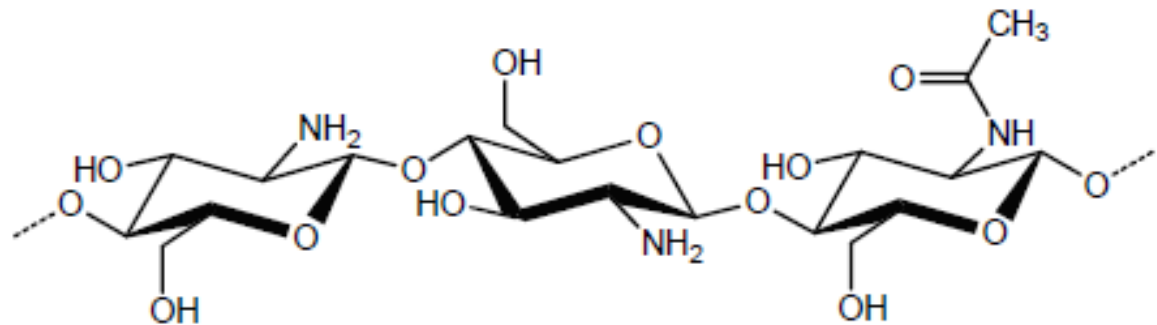

\section{Wound healing}

Since discovery, chitosan has been widely used as a topical dressing in wound management since it is homeostatic, stimulates healing, has antimicrobial potential, nontoxic, biocompatible and biodegradable (Dai et al., 2011). Based on these, several studies have been performed on them.

Burkatovskaya et al., (2006) compared the antimicrobial ability of chitosan acetate bandage called as HemCom ${ }^{\mathrm{TM}}$ bandage with alginate sponge bandage and silver sulfadiazine cream in mouse models of infected open wounds. Ong et al., (2008) used chitosan dressing by incorporating polyphosphate and silver for improved hemostatic and antimicrobial effects. Caetano et al., (2014) investigated the healing properties of a chitosan-alginate membrane on a cutaneous wound in rats and found improving effects in the process of wound healing. Howling et al., (2001) showed that chitosan enhanced fibroblast proliferation, and found that the proliferative effect is related to the deacetylation degree of the chitosan. The samples with a high deacetylation degree i.e. $84 \%$ and $86 \%$ deacetylation degree was found to have increased the mitogenic activity in fibroblasts, while some sample of chitin was found to have antiproliferative effect. Some commercial hydrogels of chitosan such as Chitipack $\mathrm{P} \circledast$ Eisai Co, Tegasorb ${ }^{\circledR} 3 \mathrm{M}$, Chitoseal ${ }^{\circledR}$ Abbott etc was found to be used as functionalized wound healing dressing and also in drug targeting.

These chitosan based hydrogels with modifications was found to provide a moist wound environment, protects the wound from secondary infections, remove wound exudates, promotes re-epithalization of damaged skin, accelerates angiogensis and aids in collagen maturity (Liu et al., 2018). The effect of chitosan in combination with propolis was found to show better wound healing activity in rats (Abbaszadeh et al., 2019). 


\section{Drug delivery system}

Drug delivery systems essentially comprises of one or more pharmacologically active agent with a suitable carrier (Smolen 1978). The main criteria of ideal delivery systems are to deliver the appropriate amount of active agent at a constant/suitable rate for a pre-determined period of time and should able to channel the active agent solely to the site of action in the body (Coelho et al., 2010; Cirillo et al., 2014).

Chitosan is one such agent that carries the actual drug to the target site and perform its function with no compromises. Therefore, they have been studied for many of their drug targeting activity.

The hydrogel films developed from chitosan and xantham gum at a ratio of 50:50 along with amoxicillin were developed into semi interpenetrating networks and they were found to have higher drug release at the target site (Monga and Wanchoo 2014). Similarly, chitosan-alginate hydrogels loaded with BMP-2 and mesenchymal stem cells (MSCs) were shown to induce subcutaneous bone formation (Park et al., 2005). Dhaliwal et al., (2008) proved the oral bioavailability of acyclovir 3-fold and 4-fold due to 266 the incorporation of this drug in chitosan and thiolated chitosan, 267 respectively. In this way, chitosan plays a major role as drug targeting systems.

Chitosan is one of the functional and natural polysaccharide with more biomedical applications. With several modifications like synthesizing them as nanoparticles, using as combinations with other biological materials and drugs will make them function much better for many of our medical ailments. From the present review, it has been established that chitosan has its long run in the field of biomedicine and further research on them will get benefits more from them.

\section{References}

Abbaszadeh, A., A. Rajabzadeh and 1. Zarei (2019). Effect of Chitosan/Propolis Biodegradable Film on Full-Thickness Wound Healing in Rats. Iranian Journal of Veterinary Surgery, 14(1), 9-17.

Abdelghany, A. M., A. A. Menazea and A. M. Ismail (2019). Synthesis, characterization and antimicrobial activity of Chitosan/Polyvinyl Alcohol blend doped with Hibiscus Sabdariffa L. extract. Journal of Molecular Structure, 1197, 603-609.

Acharya, B., V. Kumar, M. C. Varadaraj, R. Lalitha and N. Rudrapatnam (2005). Characterization of chitooligosaccharides prepared by chitosanolysis with the aid of papain and pronase, and their bactericidal action against Bacillus cereus and $\mathrm{E}$. coli. Biochemical Journal, 391, 167 175.

Alamry, K. A., M. A. Hussein, Y. O. AlGhamdi, T. S. Saleh, A. M. Asiri and A. M. Alhebshi (2018). Potential anticancer performance of chitosan-based $\beta$-ketosulfone derivatives. Cogent Chemistry, 4(1), 1559435.

$\mathrm{Au}$ Natural Herbals. Chitosan history. Available from: URL: http://www.chitosan-

weightloss.net/history.html. Accessed November 22, 2001.

Avelelas, F., A. Horta, L. F. Pinto, S. C. Marques, P. M. Nunes, P. Pedrosa and S. M. Leandro (2019). Antifungal and antioxidant properties of chitosan polymers obtained from nontraditional Polybius henslowii sources. Marine drugs, 17(4), 239.

Badawy, M. E., E. I. Rabea, N. E. Taktak and M. A. El-Nouby (2016). The antibacterial activity of chitosan products blended with monoterpenes 
and their biofilms against plant pathogenic bacteria. Scientifica.

Balmayor, E. R., E. T. Barana, H. S. Azevedoa and R. L. Reisa (2011). Injectable biodegradable starch/chitosan delivery system for the sustained release of gentamicin to treat bone infections. Carbohydrate polymer 2011 87, 32-39.

Benhabiles, M. S., R. Salah, H. Lounici, N. Drouiche, M. F. A. Goosen and N. Mameri (2012). Antibacterial activity of chitin, chitosan and its oligomers prepared from shrimp shell waste. Food hydrocolloids, 29(1), 4856.

Burkatovskaya, M., G. P. Tegos and E. Swietlik (2006). Use of chitosan bandage to prevent fatal infections developing from highly contaminated wounds in mice. Biomaterials. 27(22): 4157-4164.

Caetano, G., M. Frade, T. Andrade, M. Leite, C. Zorzi Bueno, A. Moraes and J. T. Ribeiro-Paes (2014). Chitosan-alginate membranes accelerate wound healing. J. Biomed. Mater. Res. B., 103(5): 1013-1022.

Chung, Y., Y. Su, C. Chen, G. Jia, H. Wang and J. Wu (2004). Relationship between antibacterial activity of chitosan and surface characteristics of cell wall. Acta Pharmacologica Sin, 25, 932-936.

Cirillo, G., S. Hampel, U. G. Spizzirri, O.I. Parisi, N. Picci and F. Iemma (2014). Carbon nanotubes hybrid hydrogels in drug delivery: a perspective review. BioMed Res Int. https ://doi.org/10.1155/2014/82501 7

Coelho, J. F., P. C. Ferreira, P. Alves, R. Cordeiro, A. C. Fonseca, J. R. Góis and M. H. Gil (2010). Drug delivery systems: advanced technologies potentially applicable in personalized treatments. EPMA J 1:164-209.
Dai, T., M. Tanaka, Y. Y. Huang and M. R. Hamblin (2011). Chitosan preparations for wounds and burns: antimicrobial and wound-healing effects. Expert review of anti-infective therapy, 9(7), 857-879.

De Alvarenga, E.S. (2011). Characterization and properties of chitosan. Biotechnology of biopolymers, 91, 48-53.

Dhaliwal, S., S. Jain, H. Singh and A. Tiwary (2008). Mucoadhesive microspheres for gastroretentive delivery of acyclovir: in vitro and in vivo evaluation, AAPS J., 10: 322-330.

Dutta, Pradipdutta, Joydeep and Tripathi, V. (2003). Chitin and chitosan: Chemistry, properties and applications. J. Sci. Indus. Res. 63.

Goy, R. C., S. T. Morais and O.B. Assis (2016). Evaluation of the antimicrobial activity of chitosan and its quaternized derivative on $E$. coli and $S$. aureus growth. Revista Brasileira de Farmacognosia, 26(1), 122-127.

Hirano, S. (1997). N-acyl, N-arylidene- and $\mathrm{N}$-alkylidene chitosans, and their hydrogels, Chitin handbook, edited by R A A Muzzarelli and M G Peter, (European Chitin Society, Italy), 7176.

Howling, G.I., P. W. Dettmar, P. A. Goddard, F. C. Hampson, M. Dornish and E. J. Wood (2001). The effect of chitin and chitosan on the proliferation of human skin fibroblasts and keratinocytes in vitro. Biomaterials., 22, 2959-2966.

Je, J. and S. Kim (2006). Chitosan derivatives killed bacteria by disrupting the outer and inner membrane. Journal of Agricultural Food Chemistry, 54, 6629-6633.

Khanafari, A., R. Marandi and S. H, Sanatei (2008). Recovery of chitin and chitosan from shrimp waste by chemical and microbial methods. 
Iranian Journal of Environmental Health Science and Engineering, 5(1), 19-24.

Kinsella, J. E., E. Frankel, B. German, B and J. Kanner (1993). Possible mechanisms for the protective role of antioxidants in wine and plant foods. Food technology (Chicago), 47(4), 85-89.

Kumar, M. N. V. R. (2000). A review of chitin and chitosan applications. Reactive \& Functional Polymers., 46, 1-27.

Kurita, K. (1998). Chemistry and application of chitin and chitosan. Polymer Degradation and stability, 59(1-3), $117-120$.

Li, Q., L. Wei, J. Zhang, G. Gu and Z. Guo (2019). Significantly enhanced antioxidant activity of chitosan through chemical modification with coumarins. Polymer Chemistry, 10(12), 1480-1488.

Limam, Z., S. Selmi, S. Sadok, and A. El-abed (2011). Extraction and characterization of chitin and chitosan from crustacean by-products: biological and physicochemical properties. African Journal of Biotechnology, 10(4), 640647.

Liu, H., C. Wang, C. Li, Y. Qin, Z. Wang, F. Yang and J. Wang (2018). A functional chitosan-based hydrogel as a wound dressing and drug delivery system in the treatment of wound healing. RSC advances, 8(14), 75337549.

Liu, X., L. Yun, Z. Dong, L. Zhi and D. Kang (2001). Antibacterial action of chitosan and carboxymethylated chitosan. Journal of Applied Polymers Science, 79(7), 1324-1335.

Monga, T. S and R. K. Wanchoo (2014). Sorption and drug release studies from semi-interpenetrating polymer networks of chitosan and xanthan gum. Chem. Biochem. Eng., 28:105-115.

Ong, S. Y, J. Wu and S. M. Moochhala
(2008). Development of a chitosanbased wound dressing with improved hemostatic and antimicrobial properties. Biomaterials. 29(32):43234332.

Park, D.J., B. H. Choi, S. J. Zhu, J. Y. Huh, B. Y. Kim and S. H. Lee (2005). Injectable bone using chitosan alginate gel/mesenchymal stem cells/BMP-2 composites, J. Cranio-maxillo-facial Surg., 33: 50-54.

Qi, L. and Z. Xua (2006). In vivo antitumor activity of chitosan nanoparticles. Bioorganic \& Medicinal Chemistry Letters 16, 4243-4245

Qi, L. F., Z. R. Xu, Y. Li, X. Jiang and X. Y. Han (2005). In vitro effects of chitosan nanoparticles on proliferation of human gastric carcinoma cell line MGC803 cells. World Journal of Gastroenterology: 11(33), 5136.

Rajalakshmi, A., N. Krithiga and A. Jayachitra (2013). Antioxidant activity of the chitosan extracted from shrimp exoskeleton. Middle East J. Sci. Res, 16(10), 1446-1451.

Sashiwa, H. and Y. Shigemasa (1999). Chemical modification of chitin and chitosan 2: preparation and water soluble property of Nacylated or Nalkylated partially deacetylated chitins, Carbohydr. Polym., 39 (1999) 127.

Smolen, V. F (1978). Bioavailability and pharmacokinetic analysis of drug responding systems. Annu. Rev. Pharmacol. Toxicol., 18:495-522.

Sousa, F., G. M. Guebitz and V. Kokol (2009). Antimicrobial and antioxidant properties of chitosan enzymatically functionalized with flavonoids. Process Biochemistry, 44(7), 749-756.

Tan, G., M. Kaya, A. Tevlek, I. Sargin and T. Baran (2018). Antitumor activity of chitosan from mayfly with comparison to commercially available low, medium and high molecular weight 
chitosans. In Vitro Cellular \& Nanotechnology, 7(1), 1861-1870. Developmental Biology- Zimoch-Korzycka, A., L. Bobak and A. Animal, 54(5), 366-374. Jarmoluk (2016). Antimicrobial and

Zhang, H., F. Wu, Y. Li, X. Yang, J. Huang and G. Liu (2016). Chitosan-based nanoparticles for improved anticancer efficacy and bioavailability of mifepristone. Beilstein Journal of antioxidant activity of chitosan/hydroxypropyl methylcellulose film-forming hydrosols hydrolyzed by cellulase. Int. J Mol. Sci., 17(9), 1436.

\section{How to cite this article:}

Sundaramoorthy, N., G. Ramya, A. Lathifa Thasneem, D. Kaviarasi, J. Hemapriya, R. Aswini and Vijayanand, S. 2021. Biomedical Applications of Chitosan and its Derivatives - A Review. Int.J.Curr.Microbiol.App.Sci. 10(01): 3636-3643. doi: https://doi.org/10.20546/ijcmas.2021.1001.429 